\title{
Whole-genome sequence analyses of Glaesserella parasuis isolates reveals extensive genomic variation and diverse antibiotic resistance determinants
}

\author{
Xiulin Wan ${ }^{1}$, Xinhui $\mathrm{Li}^{2}$, Todd Osmundson ${ }^{3}$, Chunling Li ${ }^{\text {Corresp., } 4}$, He Yan ${ }^{\text {Corresp. } 1}$ \\ ${ }^{1}$ School of Food Science and Engineering, South China University of Technology, Guangzhou, China \\ 2 Department of Microbiology, University of Wisconsin-La Crosse, La Crosse, United States \\ 3 Department of Biology, University of Wisconsin-La Crosse, La Crosse, United States \\ 4 Institute of Animal Health Guangdong Academy of Agricultural Sciences, Guangzhou, China \\ Corresponding Authors: Chunling Li, He Yan \\ Email address: Iclclare@163.com, yanhe@scut.edu.cn
}

Background: Glaesserella parasuis (G. parasuis) is a respiratory pathogen of swine and the etiological agent of Glässer's disease. The structural organization of genetic information, antibiotic resistance genes, potential pathogenicity, and evolutionary relationships among global G. parasuis strains remain unclear. The aim of this study was to better understand patterns of genetic variation, antibiotic resistance factors, and virulence mechanisms of this pathogen.

Methods: The whole-genome sequence of a ST328 isolate from diseased swine in China was determined using Pacbio RS II and Illumina MiSeq platforms and compared with 54 isolates from China sequenced in this study and 39 strains from China and eight other countries sequenced by previously. Patterns of genetic variation, antibiotic resistance, and virulence mechanisms were investigated in relation to the phylogeny of the isolates. Electrotransformation experiments were performed to confirm the ability of pYL1 - a plasmid observed in ST328 - to confer antibiotic resistance.

Results: The ST328 genome contained a novel Tn6678 transposon harbouring a unique resistance determinant. It also contained a small broad-host-range plasmid pYL1 carrying aac(6')-le-aph(2")-la and bla $_{\mathrm{ROB}-1}$; when transferred to Staphylococcus aureus RN4220 by electroporation, this plasmid was highly stable under kanamycin selection. Most (85.13-91.74\%) of the genetic variation between G. parasuis isolates was observed in the accessory genomes. Phylogenetic analysis revealed two major subgroups distinguished by country of origin, serotype, and multilocus sequence type (MLST). Novel virulence factors ( $g i g P, m a l Q$, and gmhA) and drug resistance genes (norA, bacA, ksgA, and bcr) in G. parasuis were identified. Resistance determinants (sul2, aph(3")-Ib, norA, bacA, ksgA, and bcr) were widespread across isolates, regardless of serovar, isolation source, or geographical location.

Conclusions: Our comparative genomic analysis of worldwide G. parasuis isolates provides valuable insight into the emergence and transmission of $G$. parasuis in the swine industry. The result suggests the importance of transposon-related and/or plasmid-related gene variation in the evolution of G. parasuis. 
4

6

7

8 genomic variation and diverse antibiotic resistance determinants

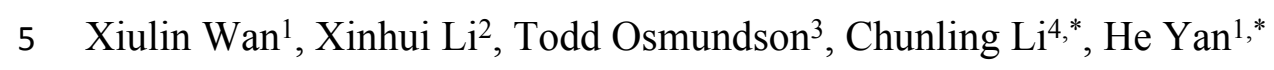
Guangdong, China WI 54601, USA 54601, USA Guangdong, China

*Corresponding author:

He Yan

381 Wushan Road, Tianhe District, Guangzhou, Guangdong, 510641, China

E-mail address: yanhe@scut.edu.cn

Chunling Li

E-mail address: 1clclare@163.com

†These authors have contributed equally to this work.

\section{Whole-genome sequence analyses of Glaesserella parasuis isolates reveals extensive}

${ }^{1}$ School of Food Science and Engineering, South China University of Technology, Guangzhou,

${ }^{2}$ Department of Microbiology, University of Wisconsin-La Crosse, 1725 State Street, La Crosse,

${ }^{3}$ Department of Biology, University of Wisconsin-La Crosse, 1725 State Street, La Crosse, WI

4 Institute of Animal Health Guangdong Academy of Agricultural Sciences, Guangzhou,

21 Baishigang Street, Wushan Road, Tianhe District, Guangzhou, Guangdong, 510640, China 
24 Abstract:

25

Background: Glaesserella parasuis (G. parasuis) is a respiratory pathogen of swine and the etiological agent of Glässer's disease. The structural organization of genetic information, antibiotic resistance genes, potential pathogenicity, and evolutionary relationships among global G. parasuis strains remain unclear. The aim of this study was to better understand patterns of genetic variation, antibiotic resistance factors, and virulence mechanisms of this pathogen.

Methods: The whole-genome sequence of a ST328 isolate from diseased swine in China was determined using Pacbio RS II and Illumina MiSeq platforms and compared with 54 isolates from China sequenced in this study and 39 strains from China and eigtht other countries sequenced by previously. Patterns of genetic variation, antibiotic resistance, and virulence mechanisms were investigated in relation to the phylogeny of the isolates. Electrotransformation experiments were performed to confirm the ability of pYL1 - a plasmid observed in ST328 - to confer antibiotic resistance.

Results: The ST328 genome contained a novel Tn6678 transposon harbouring a unique resistance determinant. It also contained a small broad-host-range plasmid pYL1 carrying aac(6')-Ie-aph(2")-Ia and bla ROB-1 ; when transferred to Staphylococcus aureus RN4220 by electroporation, this plasmid was highly stable under kanamycin selection. Most (85.13-91.74\%) of the genetic variation between G. parasuis isolates was observed in the accessory genomes. Phylogenetic analysis revealed two major subgroups distinguished by country of origin, serotype, and multilocus sequence type (MLST). Novel virulence factors (gigP, malQ, and gmhA) and drug resistance genes (norA, bacA, $k s g A$, and $b c r)$ in G. parasuis were identified. Resistance determinants (sul2, aph(3")-Ib, norA, bacA, ksgA, and bcr) were widespread across isolates, regardless of serovar, isolation source, or geographical location.

Conclusions: Our comparative genomic analysis of worldwide G. parasuis isolates provides valuable insight into the emergence and transmission of $G$. parasuis in the swine industry. The result suggests the importance of transposon-related and/or plasmid-related gene variations in the evolution of G. parasuis. 


\section{Introduction}

52

Glaesserella parasuis, a gram-negative bacterium in the family Pasteurellaceae (Dickerman et al., 2020), is a respiratory pathogen that affects swine. It is the etiological agent of Glässer's disease, which can lead to pneumonia without signs of systemic disease (Brockmeier, 2004). As China is one of the world's largest pork producers, with more than 463 million pigs accounting for approximately 50\% of global population (Zhou et al., 2013), G. parasuis outbreaks in this country could pose a significant threat to pig health and economic loss worldwide (Brockmeier et al., 2014). Disease progression and severity are influenced by virulence and antibiotic resistance, both of which can result from evolutionary processes including mutation and horizontal gene transfer (Deng et al., 2019). Although antibiotic resistance may incur fitness costs in terms of virulence, the two phenomena may also act synergistically (Geisinger and Isberg, 2017).

Antimicrobial agents are widely used to prevent and control G. parasuis infection; however, overuse of antibiotics for non-therapeutic applications - including promoting growth in healthy individuals - has resulted in the evolution of antibiotic resistant G. parasuis in farming environments (Zhao et al., 2018). Antibiotic resistance in G. parasuis is mainly conferred by a combination of transferable antibiotic resistance genes (ARGs) and multiple target gene mutations. To date, two $\beta$-lactam resistance genes ( $b l a_{\mathrm{ROB}-1}$ and $\left.b l a_{\mathrm{TEM}}\right)$, an aminoglycosideresistance gene (aac (6')-Ib-cr) and a mutation in the six copies of the 23S rRNA gene, associated with macrolide resistance, have been reported in G. parasuis (Doi and Arakawa, 2007; San et al., 2007; By Guo et al., 2012). G. parasuis strains often harbour multiple resistance genes and multi-drug resistance phenotypes, thus deterring clinical treatment.

PCR-based studies of $G$. parasuis strains have identified ARGs including tetB, aph(3")-Ib, aph(6)-Id, floR, sul1, and sul2 (Wissing et al., 2001; San et al., 2007; Zhao et al., 2018), and virulence factors including the haemolysin operon $(h h d B A)$, iron acquisition genes $(\operatorname{cir} A, \operatorname{tbp} A / B$ and $f h u A$ ), the restriction modification system $h s d S$, and genes involved in sialic acid utilization 
77

(neuraminidase $n a n H$ and sialyltransferase genes neuA, siaB and $l s g B$ ) (Martinez-Moliner et al., 2012; Costa-Hurtado and Aragon, 2013). Recently, whole-genome sequencing (WGS) has emerged as a powerful tool for predicting antibiotic resistance and pathogenic potential in $G$. parasuis. For instance, Li et al. (2013) reported two G. parasuis strains with potential resistance towards the antibiotics ciprofloxacin, trimethoprim, and penicillin, based on the presence of associated resistance genes; Nicholson et al. (2018) reported genomic differences in the toxinantitoxin systems between phenotypically distinct G. parasuis strains from Japan and Sweden; and Bello-Orti et al. (2014) noted the role of mobile genetic elements and strain-specific accessory genes in fostering high genomic diversity between pathogenic strains of the same serovar from diseased pigs in Japan, China, and the USA.

Though significant effort has been focused on exploring ARGs, virulence factors and other genetic characteristics of various $G$. parasuis strains, the structural organization of genetic information, ARGs, potential pathogenicity determinants, and evolutionary relationships among global $G$. parasuis strains remain unclear. In this study, we sequenced a multidrug-resistant isolate from diseased swine in Dongguan, China, then compared this genome sequence with those of 54 isolates from China sequenced by us and 39 strains from China and eight other countries sequenced by other researchers in order to improve our understanding of genomic diversity in $G$. parasuis and provide information for gaining better control to treat these infections.

\section{Materials \& Methods}

\section{Isolates}

The multidrug-resistant G. parasuis isolate HPS-1 examined in this study belongs to serotype 4 and was originally isolated from the lungs of a pig suffering from Glässer's disease in a commercial pig farm in Dongguan city, Guangdong province, China, in 2017. Susceptibility to 19 antimicrobial agents was determined by the disc agar diffusion method and the broth 
102

103

104

105

106

107

108

109

110

111

112

113

114

115

116

117

118

119

120

121

122

123

124

125

126

127

microdilution method (Pruller et al., 2017). The isolate was determined to be resistant to $\beta$ lactams, aminoglycosides, macrolides, quinolones, lincomycin, and sulfonamides (Table S1).

The other 54 G. parasuis isolates were obtained from diseased pigs from more than 20 geographically dispersed farms in China between November 2007 and May 2017 (Table S2). Bacteria species were identified by biochemical tests and 16S diagnostic PCR (Oliveira et al., 2001; de la Fuente et al., 2007).. All 55 G. parasuis isolates were characterised using serotyping and MLST as previously described (Wang et al., 2016; Jia et al., 2017).

\section{Genome sequencing, assembly, and bioinformatics analysis}

Isolates were cultured on tryptic soy agar or in tryptic soy broth (Oxoid, Hampshire, UK) supplemented with $10 \mathrm{mg} / \mathrm{mL}$ nicotinamide adenine dinucleotide and $5 \%$ bovine serum at $37^{\circ} \mathrm{C}$ in $5 \% \mathrm{CO}_{2}$ for $24 \mathrm{~h}$. Total genomic DNA was extracted using the DNeasy DNA extraction kit (Axygen, Union City, CA, USA).

Among the 55 isolates, one multidrug-resistant isolate (HPS-1) and one sensitive isolate (HPS-2) from diseased swine in Guangdong were randomly selected for WGS using the PacBio RSII (Pacific Biosciences, MenloPark, CA, USA) and Illumina MiSeq (Illumina, San Diego, CA, USA) platforms as previously described (Zheng et al., 2017). The genome assemblies of HPS-1 generated in this study were deposited in GenBank under accession number CP040243. The plasmid pYL1 and transposon Tn6678 of HPS-1 were submitted to GenBank under accession number MK182379 and MK994978, respectively. Genomic libraries of the other 53 genomes were generated and sequenced using the Illumina HiSeq 4000 system (Illumina, San Diego, CA, USA) as previously described (Soge et al., 2016). WGS data were assembled using SOAPdenovo v1.05 software (assembly statistics available in supplementary materials, Table S3). Gene prediction was performed using GeneMarkS (Besemer et al., 2001), and a whole-genome BLAST (Altschul et al., 1990) searches (E-value $\leq 1 \mathrm{e}^{-5}$, minimal alignment length percentage $\geq$ 80\%) against 6 databases: Kyoto Encyclopedia of Genes and Genomes (KEGG), Clusters of Orthologous Groups (COG), NCBI non-redundant protein database (NR), Swiss-Prot, Gene 
128

129

130

131

132

133

134

135

136

137

138

Ontology (GO), and TrEMBL.

\section{Phylogenetic and clustering analyses}

Two phylogenetic trees were constructed to assess the relatedness of the 55 G. parasuis strains and 39 previously published genome sequences using single-copy core orthologs and single nucleotide polymorphisms (SNPs) (Table S2). Phylogenetic inference was conducted using a maximum-likelihood optimality criterion as implemented in PhyML v3.0 (Guindon et al., 2010). The WAG amino acid substitution matrix was used for inference of the single-copy core ortholog tree, and the HKY85 nucleotide substitution model was used for inference of the SNP tree. The SNP tree was rooted using Glaesserella sp.15-184 as an outgroup. The gene contents of all 94 isolates were compared using CD-HIT (v 4.6.1) software to generate non-paralogous gene clusters (identity $\geq 0.8, \geq 80 \%$ the length of the longest cluster).

\section{Comparison of antimicrobial resistance and virulence genes}

A whole-genome BLAST search (E-value $\leq 1 \mathrm{e}^{-5}$, minimal alignment length percentage $\geq$ $80 \%$ ) was performed against four databases for pathogenicity and drug resistance analysis: Pathogen Host Interactions (PHI), Virulence Factors of Pathogenic Bacteria (VFDB), Carbohydrate-Active enZYmes Database (CAZy), and Integrated Antibiotic Resistance Genes Database (IARDB).

\section{Features of the novel Tn6678 transposon in HPS-1}

Based on the results of the BLASTn search, genomic characteristics were compared among four isolates that harboured a transposon Tn6678-like structure. BLASTn searches were performed to identify genes homologous to $b c r$, encoding the multidrug efflux system BCR/CflA, The homologuous sequences were aligned using MUSCLE algorithm in MEGA 7.0.26 (Kumar et al., 2016) and manually adjusted, yielding 92 candidate genes. The default parameter for gap opening and gap extension were used. The phylogenetic tree was generated using MEGA 7.0.26 software using the neighbour-joining method (Kumar et al., 2016) with the Kimura 2-parameter substitution model; branch support was assessed using 1000 bootstrap replicates. 
154

155

156

157

158

159

160

161

162

163

164

165

166

167

168

169

170

171

172

173

174

175

176

177

178

179

180

\section{Electrotransformation and plasmid stability test}

Plasmid pYL1 harboring two antimicrobial resistance genes, bla $a_{\mathrm{ROB}-1}$ and $a a c\left(6^{\prime}\right)-I e-$ $\operatorname{aph}\left(2^{\prime \prime}\right)-I a$, which confer to $\beta$-lactams and aminoglycosides resistance. To determine the contributions of pYL1 to penicillin and aminoglycoside antibiotic resistance, electrotransformation experiments were performed using Staphylococcus aureus RN4220 as the recipient as previously described (Wang et al., 2015). Transformants were selected on brainheart infusion (BHI) agar supplemented with kanamycin $(25 \mu \mathrm{g} / \mathrm{mL})$ for colony growth at $37^{\circ} \mathrm{C}$ for $16 \mathrm{~h}$. Transformation efficiency was calculated based on the ratio of transformants to the total number of viable cells. The presence of the $a a c\left(6^{\prime}\right)-I e-a p h\left(2^{\prime \prime}\right)-I a$ and $b l a_{\mathrm{ROB}-1}$ genes in transformants was confirmed by PCR amplification followed by DNA sequence analysis. The primers for bla $a_{\mathrm{ROB}-1}$ (494 bp) were 5'-CGCTTTGCTTATGCGTCCAC-3' (forward) and 5'ACTTTCCACGATGTTGGCGT-3'. The primers for aac(6')-Ie-aph(2')-Ia (412 bp) were 5'AGAGCCTTGGGAAGATGAAGTT-3' (forward) and 5'-TGCCTTAACATTTGTGGCATT-3' (reverse). The primers were designed using NCBI Primer-BLAST. The PCR conditions were as follows: initial denaturation at $95{ }^{\circ} \mathrm{C}$ for $5 \mathrm{~min}, 30$ cycles of amplification $\left(30 \mathrm{~s}\right.$ at $95{ }^{\circ} \mathrm{C}, 30 \mathrm{~s}$ at $58{ }^{\circ} \mathrm{C}$, and $90 \mathrm{~s}$ at $72{ }^{\circ} \mathrm{C}$ ), followed by extension at $72{ }^{\circ} \mathrm{C}$ for $10 \mathrm{~min}$. The PCR products were purified and sequenced by Majorbio Company (Shanghai, China). The MICs of S. aureus RN4220 and five transformants were determined by Etest (Liofilchems.r.1.) according to the manufacturer's instructions.

The stability of plasmids carrying $a a c\left(6^{\prime}\right)-I e-a p h\left(2^{\prime \prime}\right)-I a$ and $b l a_{\mathrm{ROB}-1}$ was determined by serial passages for 15 consecutive days at 1:1000 dilutions into fresh BHI, with or without antibiotic (kanamycin) pressure. Serially diluted cultures were spread on BHI agar plates with or without kanamycin $(8 \mu \mathrm{g} / \mathrm{mL})$, and the resistance retention rate was determined by randomly picking at least 50 colonies from the BHI plates, spotting them onto new BHI plates with kanamycin $(8 \mu \mathrm{g} / \mathrm{mL})$, and calculating the ratio of resistant to total colonies. Both the resistant and susceptible colonies from the plates were randomly picked and subjected to PCR for detection of bla $a_{\mathrm{ROB}-1}$ and $a a c\left(6^{\prime}\right)-I e-\operatorname{aph}\left(2^{\prime \prime}\right)-I a$. 
181

182

183

184

185

186

187

188

189

190

191

192

193

194

195

196

197

198

199

200

201

202

203

204

\section{Results}

\section{G. parasuis core and unique genes}

Compilation of the 94 genomes covering all serovars and disease- and non-disease-causing backgrounds from nine geographic locations (Table S2) demonstrated expansion of the pangenome, whereas the number of core genes remained relatively stable with the addition of new strains (Fig. 1A). This result suggests the presence of an open pan-genome experiencing frequent evolutionary changes through gene gains and losses or lateral gene transfer. The size of the pangenome was 5,243 genes, including $\sim 3.34 \%$ core genes shared among the 94 isolates mainly from China (Fig. 1B). Meanwhile, accessory genomes occupied a large fraction (85.13-91.74\%) of the G. parasuis gene content compared with the core genomes and the number of unique genes ranged from 0 to 103 indicating that $0-4.6 \%$ of the genome consists of strain-specific accessory genes (Table S4).

Clusters of Orthologous Groups classification indicated that core genes were significantly enriched in defense mechanisms and inorganic ion transport and metabolism, whereas unique genes were significantly enriched in unknown function, nucleotide transport and metabolism, and carbohydrate transport and metabolism (Fig. 1C).

\section{Phylogenetic analysis of $G$. parasuis isolates}

A phylogenetic tree based on single-copy core genes of our isolates and reference isolates resolved two well-supported lineages, lineages I and II, exhibiting association with country, serotypes, and MLST types (Fig. 2). Lineages I and II comprised eight and two countries, respectively. Serovars 5, 12, and 14 were identified predominantly in lineage I, while serovars 2 and 10 were mostly found in lineage II. For serovars 3, 8, 9, and 11, the numbers of isolates were too low to draw conclusions about phylogenetic patterns. The remainder of the serovars were found in both clades. 
MLST analysis assigned the 39 isolates in GenBank to 20 different STs, including six new

206

207

208

209

210

211

212

213

214

215

216

217

218

219

220

221

222

223

224

225

226

227

228

229

230

STs, with 13 isolates not determined. The 55 isolates obtained in our study belonged to 49 different STs, including 39 new STs (Table S2). Most strains of the same STs formed single clades (Fig. 2). The SNP-based tree with and without an outgroup (Fig. S1 and Fig. S2) was consistent with the phylogenetic analysis based on single-copy core orthologs. The number of whole-genome SNP differences among the 94 isolates ranged from 8,603 to 8,730.

\section{Biological features of $G$. parasuis isolates}

Variation in virulence and stress resistance genes was observed among G. parasuis lineages and subgroups (Fig. 3). All 94 G. parasuis isolates harboured more than five types of pathogenic factors. The virulence factors gigP, malQ, and $g m h A$ were carried by all the tested G. parasuis isolates. Moreover, other virulence factors including the $r f a$ cluster, encoding enzymes for lipopolysaccharide (LPS) core biosynthesis, and galU and galE, resulting in impaired biofilm formation, were universally present in the G. parasuis isolates.

The main ARGs associated with resistance in G. parasuis, including the $\beta$-lactam-resistant gene $b l a_{\mathrm{ROB}-1}$, tetracycline resistance genes $t e t B$, aminoglycoside resistance genes $a p h\left(3^{\prime \prime}\right)-I b$ and $\operatorname{aac}\left(6^{\prime}\right)-I e-a p h\left(2^{\prime \prime}\right)-I a$, fluoroquinolone resistance gene nor $A$, chloramphenicol resistance genes catIII and floR, sulfonamide resistance gene sul2 were discovered (Fig. 3). Among all of these genes, the genes sul2 and $a p h\left(3^{\prime \prime}\right)-I b$, and $\beta$-lactam-resistant genes $p b p 1 a$ and $p b p 3 a$ were universally present in the G. parasuis isolates (Fig. 3). Three different serotype isolates (H82, H92, and H313) obtained from different sites in different years that clustered closely in one branch all harboured the lincosamide antibiotic resistance factor lunC (Fig. 3). Moreover, 91.5\% of the isolates had $b c r, 90.42 \%$ of the isolates had $b a c A, 100 \%$ of the isolates had $k s g A$, but five isolates had norA.

\section{Genomic features of $G$. parasuis HPS-1}

Following sequencing and assembly, a 2,326,414-bp chromosome with an average $\mathrm{G}+\mathrm{C}$ content of $40.03 \%$, and a 7,777-bp small plasmid sequence (pYL1) with an average $\mathrm{G}+\mathrm{C}$ content 
231 of 33.32\% were identified in strain HPS-1 (Supplementary Fig. S3 and Fig. 4). HPS-1 exhibited 232 a novel ST (ST328) with undescribed MLST alleles or previously unreported allelic 233 combinations. This ST328 genome harbored resistance genes against several types of antibiotics,

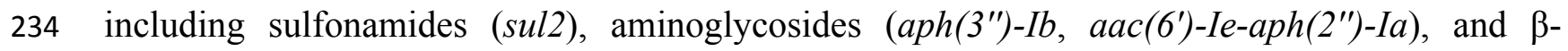
235 lactam $\left(b l a_{\mathrm{ROB}-1}\right)$ (Table S1). Further, this genome contained efflux pump-related genes that 236 confer resistance to sulfonamides $(b c r)$ and multidrug resistance $(a c r B)$.

237 We also identified a novel transposon in the ST328 isolate, designated Tn6678 in the Tn 238 Number Registry (https://transposon.lstmed.ac.uk/). This transposon harbours two 966-bp IS110 239 family transposases at both ends, two toxin genes pilT and phd, two genes associated with the 240 two-component signal transduction system cpxA and $\operatorname{cpx} R$, one efflux pump-associated gene $b c r$, 241 and four genes encoding hypothetical proteins with unknown function (Fig. 5). Genome analysis 242 revealed that Tn6678 was inserted between the molybdopterin molybdotransferase MoeA 243 encoded by moeA and 3-isopropylmalate dehydratase large subunit encoded by leuC. A LacI 244 family transcriptional regulator and a bifunctional tRNA (5-methylaminomethyl-2245 thiouridine)(34)-methyltransferase MnmD/FAD-dependent 5-carboxymethylaminomethyl-2246 thiouridine (34) oxidoreductase MnmC flanked the transposon to the right and left, respectively.

Through BLASTN searches, highly conserved homologous sequences to Tn 6678 (>97\% nucleotide sequence similarity) were identified in four G. parasuis strains [29755 (GenBank accession number CP021644, USA), SH0165 (CP001321, China), ZJ0906 (CP005384, China), and str. Nagasaki (NZ_APBT00000000, Japan)]. The only differences in these five chromosomes were in the transposases, but transposon Tn6678 had two complete inverted repeats of IS110 transposases flanked by 32-bp inverted repeats of ISNme5 at both ends (Fig. 5), suggesting mobility potential. The bcr-containing Tn6678 also contained an antibiotic resistance gene cassette, suggesting its potential to transfer antibiotic resistance genes.

BLASTn searches for the $b c r$ gene returned a large set of divergently related sequences using default parameters. These sequences were annotated as bicyclomycin/multidrug efflux system, Bcr/CflA family drug resistance efflux transporter, Bcr/CflA family multidrug efflux 
258

259

260

261

262

263

264

265

266

267

268

269

270

271

272

273

274

275

276

277

278

279

280

281

282

283

major facilitator superfamily (MFS) transporter or drug resistance transporter, and Bcr/CflA subfamily. Phylograms revealed that the bcr gene in HPS-1 was most closely related to homologs identified in other members of the Pasteurellaceae, particularly G. parasuis, Actinobacillus indolicus, Bibersteinia trehalosi, Actinobacillus (A. pleuropneumoniae, A. suis, A. equuli, A. lignieresii, A. indolicus, and A. porcitonsillarum), and Mannheimia (M. haemolytica and $M$. varigena), all of which are known causative agents of upper respiratory tract infections (Fig. 6).

The neighbour-joining phylogenetic tree using 92 bcr genes selected from the BLASTn searches clearly demonstrated two distinctive clades. The first clade contained $b c r$ genes of Hemophilus influenzae, which colonizes humans, and other Haemophilus species that colonize non-human animals. Members of the second clade were divided into four apparent subclades, including G. parasuis, B. trehalosi, Actinobacillus spp., and Mannheimia spp. Except for G. parasuis, the chromosomally encoded Bcr/CflA from G. parasuis HPS-1 most closely clustered with that found in A. indolicus. The phylogenetic tree indicated a divergent evolutionary pattern between animal-origin Pasteurellaceae bacteria. The bcr gene tree is consistent with the organismal phylogeny, suggesting that horizontal gene transfer does not play an important role in the evolution of $b c r$-mediated resistance.

\section{General features and electrotransformation of the plasmid pYL1}

The plasmid pYL1 identified in HPS-1 contained seven ORFs with an average length of 912 bp, with one encoded protein of undetermined function (Fig. 4), and two antimicrobial resistance genes, bla $a_{\mathrm{ROB}-1}$ and $a a c\left(6^{\prime}\right)-I e-a p h\left(2^{\prime \prime}\right)-I a$. Four ORFs were identified to encode a $3^{\prime}$-truncated transposase protein ISApl1 (30 amino acids), a Rep-like protein (444 amino acids) involved in plasmid replication, and two Mob proteins, MobC (144 amino acids) and MobA (541 amino acids), associated with plasmid mobilization (Fig. 4). Except for resistance genes, pYL1 had the same backbone and genetic structure and showed 100\% nucleotide identity to four previouslyidentified plasmids, pFZ51, pFS39, pHN61, and pHB0503 (Table S5) (Kang et al., 2009; Chen 
284 et al., 2010; Yang et al., 2013). In contrast, the resistance genes and flanking regions in pYL1 285 exhibited as little as 58\% sequence identity to the other four plasmids (Fig. 7).

286 Transformation of pYL1 into S. aureus RN4220 was achieved at a frequency of $10^{-9}$ cells 287 per recipient cell by electroporation, confirming that pYL1 is a mobilizable plasmid with active

288

289

290

291

292

293

294

295

296

297

298

299

300

301

302

303

304

305

306

307

308

mobilization genes. The transformants had increased MICs for oxacillin, gentamicin, amikacin, kanamycin, and streptomycin as compared with those of the parental strain $(0.047$ to $>256 \mathrm{mg} / \mathrm{L}$, 0.094 to $1.5 \mathrm{mg} / \mathrm{L}, 0.38$ to $16 \mathrm{mg} / \mathrm{L}, 0.38$ to $32 \mathrm{mg} / \mathrm{L}$, and $<0.25$ to $32 \mathrm{mg} / \mathrm{L}$, respectively). This finding indicated that plasmid pYL1 carrying bla $a_{\mathrm{ROB}-1}$ and $a a c\left(6^{\prime}\right)-I e-a p h\left(2^{\prime \prime}\right)-I a$ contributed to the penicillin resistance and aminoglycoside antibiotic resistance in S. aureus RN4220 transformants. Furthermore, the plasmid showed low stability in S. aureus without antibiotic pressure, as only $52.5 \%, 30.48 \%$, and $2.68 \%$ of transformants maintained the kanamycin resistance after five, six, and seven subcultures, respectively. However, the plasmid can be conserved in S. aureus cultured with kanamycin, as $100 \%$ of the colonies remained resistant to kanamycin after 10 subcultures, as confirmed by PCR mapping.

\section{Discussion}

In the current study, we observed an open pan-genome. Similar result that the size of pangenome was 7,431 genes including 1,049 core genes has been reported (Howell et al., 2014). This suggested that the G. parasuis pan-genome is vast, and unique genes can be continuously be identified upon sequencing more G. parasuis genomes. However, the isolates in this study with $\sim 3.34 \%$ core genes, primarily isolated from China, displayed further diversity and higher variability than isolates with only $\sim 14 \%$ core genes, primarily obtained from the UK (Howell et al., 2014). Besides, we identified 54 new STs enriching the G. parasuis MLST databases and highlight the wide distribution of G. parasuis strains. Although most strains of the same STs formed single clades, there was no definitive association between ST and serotype (Fig. 2), consistent with previous studies (Olvera et al., 2006; Wang et al., 2016).

Peer] reviewing PDF | (2019:12:44235:1:1:NEW 7 Apr 2020) 
309

310

311

312

313

314

315

316

317

318

319

320

321

322

323

324

325

326

327

328

329

330

331

332

333 334

The pattern of the phylogenetic tree based on single-copy core genes was different from the population grouping predicted via MLST, which showed six main subgroups (Wang et al., 2016). Both phylogenetic lineages contain both Asian and North American isolates, in agreement with previous phylogenetic analyses (Howell et al., 2014; Wang et al., 2016; Dickerman et al.,2020) and supporting the hypothesis of frequent migration of isolates between geographic regions.

Five types of pathogenic factors $g i g P, \operatorname{malQ}, \operatorname{gmh} A, r f a$ and gal cluster were universally carried by $G$. parasuis isolates in this study. The $r f a F$ gene has been linked to serum resistance, adhesion, and invasion (Zhang et al., 2013); galU plays a role in autoagglutination and biofilm formation, and galE appears to affect biofilm production indirectly in G. parasuis (Zou et al., 2013). Serum resistance may play a role in the virulence of G. parasuis (Cerda-Cuellar and Aragon, 2008). However, $\operatorname{lsg} B$, previously associated with $G$. parasuis virulence potential, was predominant in six isolates (29755 and HPS9 from the USA, Nagasaki from Japan, and KL0318, SH0104, and SH0165 from China), in line with potentially virulent strains isolated from the nasal cavities of healthy pigs (Amano et al., 1996; Brockmeier et al., 2013).

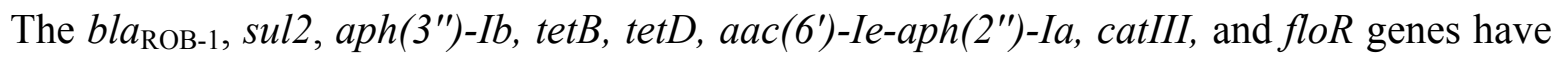
previously been identified in G. parasuis (Zhao et al., 2018). In the current study, we identified all of genes mentioned above. This is the first report of genes tetA, tet $H$ and tetR genes in $G$. parasuis isolates and needs further study. Tetracycline resistance genes are often associated with conjugative and mobile genetic elements enabling horizontal transfer (Lancashire et al., 2005; Zhao et al., 2018). Moreover, this is the first report describing the presence of the bcr, bacA, ksgA and nor $A$ genes in G. parasuis, to the best of our knowledge. All of these benefits from the application of whole genome sequencing method. Three isolates clustered closely in one branch all harboured lunC gene, contained in the ISSag10 sequence of all three isolates. The lunC gene was only identified in plasmid pHN61 of G. parasuis (Chen et al., 2010). The results suggested that the resistance of these three strains to lincomycin may be mediated by the plasmid carrying lunC gene. 
This is also the first report describing the transoson Tn6678 containing toxin genes pilT and

336

337

338

339

340

341

342

343

344

345

346

347

348

349

350

351

352

353

354

355

356

357

358

359

360

361

$p h d$, drug resistance genes cpxA and $c p x R$, and an efflux pump gene bcr. Association between the Cpx system and bacterial antimicrobial resistance has been reported in Escherichia coli, Salmonella enterica, Klebsiella pneumoniae, and G. parasuis (Hu et al., 2011; Srinivasan et al., 2012; Audrain et al., 2013; Kurabayashi et al., 2014; Cao et al., 2018). CpxR plays essential roles in mediating macrolide (i.e., erythromycin) resistance (Cao et al., 2018). The Bcr/CflA efflux system was identified as a group of antiporters that confer resistance to chloramphenicol, florfenicol, and bicyclomycin by actively transporting these compounds out of the cell (Marklevitz and Harris, 2016). The transposon Tn6678 had two complete inverted repeats of IS110 transposases flanked by 32-bp inverted repeats of ISNme5 at both ends suggesting mobility potential and its potential to transfer antibiotic resistance genes. In G. parasuis, only the efflux pump AcrB, belonging to the resistance-nodulation division (RND) family, has been analysed to date. Efflux pump AcrB may play a role in multidrug resistance, and the acr $A B$ gene cluster could affect the efflux of macrolides in G. parasuis (Feng et al., 2014). However, this is the first description of the efflux pump Bcr/CflA in G. parasuis, belonging to the MFS. This efflux pump, encoded by $b c r$, harbored on a transposon indicated its potential transferability.

To date, two $\beta$-lactam resistance genes $\left(b l a_{\mathrm{ROB}-1}\right.$ and $\left.b l a_{\mathrm{TEM}}\right)$ have been reported in $G$. parasuis (By Guo et al., 2012). A $\beta$-lactam resistance plasmid, pB1000, harbouring bla ROB-1 $_{\text {was }}$ previously detected in G. parasuis clinical strains isolated from Glässer's disease lesions (San et $a l ., 2007)$. The plasmid pYL1 harboured two antimicrobial resistance genes, bla $_{\mathrm{ROB}-1}$ and $a a c\left(6^{\prime}\right)-$ Ie-aph(2")-Ia. The ROB-1 of plasmid pYL1 had a typical size of $305 \mathrm{bp}$, in line with functionally active members of the ROB-1 family from different plasmids in Pasteurellaceae species. AAC(6')-Ie-APH(2')-Ia, the most important aminoglycoside-resistance enzyme in grampositive bacteria conferring resistance to almost all known aminoglycoside antibiotics in clinical use, also had a typical size of 479 amino acids in this family (Rouch et al., 1987). Although $a a c\left(6^{\prime}\right)-I b-c r$ is considered the most prominent aminoglycoside-resistance gene in G. parasuis (Doi and Arakawa, 2007; San et al., 2007), the bifunctional aminoglycoside-resistance enzyme 
362

363

364

365

366

367

368

369

370

371

372

373

374

375

376

377

378

379

380

381

382

383

384

AAC(6')-Ie-APH(2')-Ia in plasmids is also reported in GenBank for G. parasuis strains. Comparing with other four previously-identified plasmids which have similar structure with pYL1 suggested more rapid evolution among the resistance-associated components of these small plasmids. The transposase gene of ISApl1 in pYL1 had an internal deletion of $659 \mathrm{bp}$, but intact $3^{\prime}$ and $5^{\prime}$ ends. The truncated ISApl1 linked with bla $a_{\mathrm{ROB}-1}$ suggested that ISApl1 played a key role in transposition of $b l a_{\mathrm{ROB}-1}$, facilitating the horizontal transfer of $\beta$-lactam and aminoglycoside resistance among $G$. parasuis isolates. These results are consistent with a previous study presenting evidence for spread of $\beta$-lactam resistance (Yang et al., 2013). A similar occurrence was also identified in A. porcitonsillarum or G. parasuis plasmids pFJS5863, pQY431, and pFS39, suggesting a more widespread role and highlighting that the function of ISApl1 requires further investigation.

\section{Conclusions}

In summary, our results shed new light on the importance of genomic variations, especially transposon-related and/or plasmid-related gene variations, in the evolution of G. parasuis. This comparative analysis identified potentially novel virulence factors ( $g i g P, \operatorname{mal} Q$, and $\operatorname{gmh} A)$ and drug resistance genes (nor A, bacA, ksgA, and bcr) in G. parasuis. Resistance determinants (sul2, $\operatorname{aph}\left(3^{\prime \prime}\right)-I b$, nor $A, \operatorname{bacA}, \mathrm{ksg} A$, and $\left.b c r\right)$ were widespread across isolates, regardless of serovar, isolation source, or geographical location. Future research focused on a larger sample of $G$. parasuis isolates worldwide will further increase understanding of the rapid development of antibiotic resistance associated with mobile genetic elements in this important animal pathogen.

\section{Acknowledgements}

We thank members of our laboratories for fruitful discussions.

\section{Funding}


385

386

387

388

389

390

391

392

393

394

395

396

397

398

399

400

401

402

403

404

405

406

407

408

409

This work was supported by the National Key Basic Research Program (Grant No. 2016YFD0500606), Guangdong Provincial Key Research and Development Plan Project (Grant No. 2019B020212002), and the Central Universities constructs the world fist-class university (discipline) and Characteristic Development Guidance Special Fund (Grant No. K5174960).

\section{References}

Altschul, SF, Gish, W, Miller, W, Myers, EW and Lipman, DJ. 1990. Basic local alignment search tool. Journal of Molecular Biology, 215: 403-410 DOI: 10.1016/S0022-2836(05)80360-2.

Amano, H, Shibata, M, Kajio, N and Morozumi, T. 1996. Pathogenicity of Haemophilus parasuis serovars 4 and 5 in contact-exposed pigs. Journal of Veterinary Medical Science, 58: 559-561 DOI: 10.1292/jvms.58.559.

Audrain, B, Ferrieres, L, Zairi, A, Soubigou, G, Dobson, C, Coppee, JY, Beloin, C and Ghigo, JM. 2013. Induction of the Cpx envelope stress pathway contributes to Escherichia coli tolerance to antimicrobial peptides. Appl Environ Microbiol, 79: 7770-7779 DOI: 10.1128/AEM.02593-13.

Bello-Orti, B, Aragon, V, Pina-Pedrero, S and Bensaid, A. 2014. Genome comparison of three serovar 5 pathogenic strains of Haemophilus parasuis: insights into an evolving swine pathogen. Microbiology, 160: 1974-1984 DOI: 10.1099/mic.0.079483-0.

Besemer, J, Lomsadze, A and Borodovsky, M. 2001. GeneMarkS: a self-training method for prediction of gene starts in microbial genomes. Implications for finding sequence motifs in regulatory regions. Nucleic Acids Research, 29: 2607-2618 DOI: 10.1093/nar/29.12.2607.

Brockmeier, SL. 2004. Prior infection with Bordetella bronchiseptica increases nasal colonization by Haemophilus parasuis in swine. Veterinary Microbiology, 99: 75-78 DOI: 10.1016/j.vetmic.2003.08.013.

Brockmeier, SL, Loving, CL, Mullins, MA, Register, KB, Nicholson, TL, Wiseman, BS, Baker, RB and Kehrli, MJ. 2013. Virulence, transmission, and heterologous protection of four isolates 
410 of Haemophilus parasuis. Clinical and Vaccine Immunology, 20: 1466-1472 DOI: 411 10.1128/CVI.00168-13.

412 Brockmeier, SL, Register, KB, Kuehn, JS, Nicholson, TL, Loving, CL, Bayles, DO, Shore, SM 413 and Phillips, GJ. 2014. Virulence and draft genome sequence overview of multiple strains of the 414 swine pathogen Haemophilus parasuis. PLoSOne, 9: 103787 DOI: 415 10.1371/journal.pone.0103787.

416 Guo, LGL, Zhang, JZJ, Xu, CXC, Ren, TRT, Zhang, BZB, Chen, JCJ and Liao, MLM. 2012. 417 Detection and characterization of $\beta$-lactam resistance in Haemophilus parasuis strains from pigs 418 in South China. Journal of Integrative Agriculture, 11: 116-121 DOI.

419 Cao, Q, Feng, F, Wang, H, Xu, X, Chen, H, Cai, X and Wang, X. 2018. Haemophilus parasuis 420 CpxRA two-component system confers bacterial tolerance to environmental stresses and 421 macrolide resistance. Microbiological Research, 206: 177-185 DOI: $42210.1016 /$ j.micres.2017.10.010.

423 Cerda-Cuellar, M and Aragon, V. 2008. Serum-resistance in Haemophilus parasuis is associated 424 with systemic disease in swine. Veterinary Journal, 175: 384-389 DOI: $425 \quad 10.1016 / j . t v j 1.2007 .01 .016$.

426 Chen, LCL, Cai, XCX, Wang, XWX, Zhou, XZX, Wu, DWD, Xu, XXX and Chen, HCH. 2010. 427 Characterization of plasmid-mediated lincosamide resistance in a field isolate of Haemophilus 428 parasuis. Journal of Antimicrobial Chemotherapy, 65: 2256-2258 DOI.

429 Costa-Hurtado, M and Aragon, V. 2013. Advances in the quest for virulence factors of 430 Haemophilus parasuis. Veterinary Journal, 198: 571-576 DOI: 10.1016/j.tvj1.2013.08.027.

431 Deng, Y, Xu, H, Su, Y, Liu, S, Xu, L, Guo, Z, Wu, J, Cheng, C, and Feng, J. 2019. Horizontal 432 gene transfer contributes to virulence and antibiotic resistance of Vibrio harveyi 345 based on 433 complete genome sequence analysis. BMC Genomics, 20: 761 DOI: 10.1186/s12864-019-6137-8.

434 de la Fuente, AJ, Tucker, AW, Navas, J, Blanco, M, Morris, SJ and Gutierrez-Martin, CB. 2007. 
435

436

437

438

439

440

441

442

443

444

445

446

447

448

449

450

451

452

453

454

455

456

457

458

459

Antimicrobial susceptibility patterns of Haemophilus parasuis from pigs in the United Kingdom and Spain. Veterinary Microbiology, 120: 184-191 DOI: 10.1016/j.vetmic.2006.10.014.

Dickerman, A, Bandara, AB, and Inzana, TJ. 2020. Phylogenomic analysis of Haemophilus parasuis and proposed reclassification to Glaesserella parasuis, gen. nov., comb. nov. International Journal of Systematic and Evolutionary Microbiology, 70: 180-186. DOI: 10.1099/ijsem.0.003730.

Doi, Y and Arakawa, Y. 2007. 16S ribosomal RNA methylation: emerging resistance mechanism against aminoglycosides. Clinical Infectious Diseases, 45: 88-94 DOI: 10.1086/518605.

Feng, S, Xu, L, Xu, C, Fan, H, Liao, M and Ren, T. 2014. Role of acrAB in antibiotic resistance of Haemophilus parasuis serovar 4. Veterinary Journal, 202: 191-194 DOI: 10.1016/j.tvj1.2014.05.045.

Geisinger, E, and Isberg, RR. 2017. Interplay between antibiotic resistance and virulence during disease promoted by multidrug-resistant bacteria. Journal of Infectious Diseases, 215: S9-S17 DOI: 10.1093/infdis/jiw402.

Guindon, S, Dufayard, JF, Lefort, V, Anisimova, M, Hordijk, W and Gascuel, O. 2010. New algorithms and methods to estimate maximum-likelihood phylogenies: assessing the performance of PhyML 3.0. Systematic Biology, 59: 307-321 DOI: 10.1093/sysbio/syq010.

Howell, KJ, Weinert, LA, Chaudhuri, RR, Luan, SL, Peters, SE, Corander, J, Harris, D, Angen, O, Aragon, V, Bensaid, A, Williamson, SM, Parkhill, J, Langford, PR, Rycroft, AN, Wren, BW, Holden, MT, Tucker, AW and Maskell, DJ. 2014. The use of genome wide association methods to investigate pathogenicity, population structure and serovar in Haemophilus parasuis. BMC Genomics, 15: 1179 DOI: 10.1186/1471-2164-15-1179.

Hu, WS, Chen, HW, Zhang, RY, Huang, CY and Shen, CF. 2011. The expression levels of outer membrane proteins STM1530 and OmpD, which are influenced by the CpxAR and BaeSR twocomponent systems, play important roles in the ceftriaxone resistance of Salmonella enterica 
460

461

462

463

464

465

466

467

468

469

470

471

472

473

474

475

476

477

478

479

480

482

483

484

481 Marklevitz, J and Harris, LK. 2016. Prediction driven functional annotation of hypothetical

serovar Typhimurium. Antimicrob Agents Chemother, 55: 3829-3837 DOI: 10.1128/AAC.00216-11.

Jia, AJA, Zhou, RZR, Fan, HFH, Yang, KYK, Zhang, JZJ, Xu, YXY, Wang, GWG and Liao, MLM. 2017. Development of serotype-specific PCR assays for typing of Haemophilus parasuis isolates circulating in southern China. Journal of Clinical Microbiology, 55: 3249-3257 DOI: 10.1128/JCM.00688-17.

Kang, M, Zhou, R, Liu, L, Langford, PR and Chen, H. 2009. Analysis of an Actinobacillus pleuropneumoniae multi-resistance plasmid, pHB0503. Plasmid, 61: 135-139 DOI: 10.1016/j.plasmid.2008.11.001.

Kumar, S, Stecher, G and Tamura, K. 2016. MEGA7: Molecular Evolutionary Genetics Analysis version 7.0 for bigger datasets. Molecular Biology and Evolution, 33: 1870-1874 DOI: 10.1093/molbev/msw054.

Kurabayashi, K, Hirakawa, Y, Tanimoto, K, Tomita, H and Hirakawa, H. 2014. Role of the CpxAR two-component signal transduction system in control of fosfomycin resistance and carbon substrate uptake. Journal of Bacteriology, 196: 248-256 DOI: 10.1128/JB.01151-13.

Lancashire JF, Terry TD, Blackall PJ, Jennings MP. 2005. Plasmid-encoded TetB tetracycline resistance in Haemophilus parasuis. Antimicrob Agents Chemother, 49: 1927-1931 DOI: 10.1128/AAC.49.5.1927-1931.2005

Li, Y, Kwok, AH, Jiang, J, Zou, Y, Zheng, F, Chen, P, Hou, C, Leung, FC and Jiang, P. 2013. Complete genome analysis of a Haemophilus parasuis serovar 12 strain from China. PLoS One, 8: e68350 DOI: 10.1371/journal.pone.0068350. proteins in the major facilitator superfamily of $S$. aureus NCTC 8325. Bioinformation, 12: 254262 DOI: 10.6026/97320630012254.

Martinez-Moliner, V, Soler-Llorens, P, Moleres, J, Garmendia, J and Aragon, V. 2012. 
485

486

487

488

489

490

491

492

493

494

495

496

497

498

499

500

501

502

503

504

505

506

507

508 509

Distribution of genes involved in sialic acid utilization in strains of Haemophilus parasuis. Microbiology, 158: 2117-2124 DOI: 10.1099/mic.0.056994-0.

Nicholson, TL, Brunelle, BW, Bayles, DO, Alt, DP and Shore, SM. 2018. Comparative genomic and methylome analysis of non-virulent D74 and virulent Nagasaki Haemophilus parasuis isolates. PLoSOne, 13: e205700 DOI: 10.1371/journal.pone.0205700.

Oliveira, S, Galina, L and Pijoan, C. 2001. Development of a PCR test to diagnose Haemophilus parasuis infections. Journal of Veterinary Diagnostic Investigation, 13: 495-501 DOI: $10.1177 / 104063870101300607$.

Olvera, A, Cerda-Cuellar, M and Aragon, V. 2006. Study of the population structure of Haemophilus parasuis by multilocus sequence typing. Microbiology, 152: 3683-3690 DOI: 10.1099/mic.0.29254-0.

Pruller, S, Turni, C, Blackall, PJ, Beyerbach, M, Klein, G, Kreienbrock, L, Strutzberg-Minder, K, Kaspar, H, Meemken, D and Kehrenberg, C. 2017. Towards a standardized method for broth microdilution susceptibility testing of Haemophilus parasuis. Journal of Clinical Microbiology, 55: 264-273 DOI: 10.1128/JCM.01403-16.

Rouch, DA, Byrne, ME, Kong, YC and Skurray, RA. 1987. The aacA-aphD gentamicin and kanamycin resistance determinant of Tn4001 from Staphylococcus aureus: expression and nucleotide sequence analysis. J Gen Microbiol, 133: 3039-3052 DOI: 10.1099/00221287-13311-3039.

San, MA, Escudero, JA, Catalan, A, Nieto, S, Farelo, F, Gibert, M, Moreno, MA, Dominguez, L and Gonzalez-Zorn, B. 2007. Beta-lactam resistance in Haemophilus parasuis is mediated by plasmid pB1000 bearing bla $\mathrm{ROB}_{1}$. Antimicrob Agents Chemother, 51: 2260-2264 DOI: 10.1128/AAC.00242-07.

Soge, OO, No, D, Michael, KE, Dankoff, J, Lane, J, Vogel, K, Smedley, J and Roberts, MC. 2016. Transmission of MDR MRSA between primates, their environment and personnel at a 
510 United States primate centre. J Antimicrob Chemother, 71: 2798-2803 DOI: 10.1093/jac/dkw236.

511 Srinivasan, VB, Vaidyanathan, V, Mondal, A and Rajamohan, G. 2012. Role of the two 512 component signal transduction system CpxAR in conferring cefepime and chloramphenicol 513 resistance in Klebsiella pneumoniae NTUH-K2044. PLoSOne, 7: e33777 DOI: 514 10.1371/journal.pone.0033777.

515 Wang, L, Ma, L, Liu, Y, Gao, P, Li, Y, Li, X and Liu, Y. 2016. Multilocus sequence typing and 516 virulence analysis of Haemophilus parasuis strains isolated in five provinces of China. Infection 517 Genetics and Evolution, 44: 228-233 DOI: 10.1016/j.meegid.2016.07.015.

518 Wang, XM, Li, XS, Wang, YB, Wei, FS, Zhang, SM, Shang, YH and Du XD. 2015. 519 Characterization of a multidrug resistance plasmid from Enterococcus faecium that harbours a 520 mobilized bcrABDR locus. J Antimicrob Chemother, 70: 609-611 DOI: 10.1093/jac/dku416.

521 Wissing, A, Nicolet, $\mathrm{J}$ and Boerlin, P. 2001. The current antimicrobial resistance situation in 522 Swiss veterinary medicine. Schweiz Arch Tierheilkd, 143: 503-510.

523 Yang, SS, Sun, J, Liao, XP, Liu, BT, Li, LL, Li, L, Fang, LX, Huang, T and Liu, YH. 2013. Co524 location of the erm $(\mathrm{T})$ gene and bla $\mathrm{ROB}_{-1}$ gene on a small plasmid in Haemophilus parasuis of pig 525 origin. J Antimicrob Chemother, 68: 1930-1932 DOI: 10.1093/jac/dkt112.

526 Zhang, B, Xu, C, Zhang, L, Zhou, S, Feng, S, He, Y and Liao, M. 2013. Enhanced adherence to 527 and invasion of PUVEC and PK-15 cells due to the overexpression of RfaD, ThyA and Mip in 528 the DeltaompP2 mutant of Haemophilus parasuis SC096 strain. Veterinary Microbiology, 162: 529 713-723 DOI: 10.1016/j.vetmic.2012.09.021.

530 Zhao, Y, Guo, L, Li, J, Huang, X and Fang, B. 2018. Characterization of antimicrobial resistance 531 genes in Haemophilus parasuis isolated from pigs in China. PeerJ, 6: e4613 DOI: $53210.7717 /$ peerj.4613.

533 Zheng, B, Yu, X, Xu, H, Guo, L, Zhang, J, Huang, C, Shen, P, Jiang, X, Xiao, Y and Li, L. 2017. 534 Complete genome sequencing and genomic characterization of two Escherichia coli strains co- 
535 producing MCR-1 and NDM-1 from bloodstream infection. Sci Rep, 7: 17885 DOI: $53610.1038 / \mathrm{s} 41598-017-18273-2$.

537 Zhou, LJ, Ying, GG, Liu, S, Zhang, RQ, Lai, HJ, Chen, ZF and Pan, CG. 2013. Excretion masses 538 and environmental occurrence of antibiotics in typical swine and dairy cattle farms in China. 539 Science of the Total Environment, 444: 183-195 DOI: 10.1016/j.scitotenv.2012.11.087.

540 Zou, Y, Feng, S, Xu, C, Zhang, B, Zhou, S, Zhang, L, He, X, Li, J, Yang, Z and Liao, M. 2013. 541 The role of galU and galE of Haemophilus parasuis SC096 in serum resistance and biofilm 542 formation. Veterinary Microbiology, 162: 278-284 DOI: 10.1016/j.vetmic.2012.08.006. 


\section{Figure 1}

Analysis of the core and pan-genome of $G$. parasuis isolates.

(A) Core and pan-genomic calculations in G. parasuis isolates. Each green point represents the number of genes conserved between genomes. All of the points are plotted as a function of the strain number $(x)$. The deduced pan-genome size: $P(x)=2483.54 x^{0.18}-461.72$. The height of the curve continues to increase because the pan-genome of $G$. parasuis is open. (B) Genes missing or present in G. parasuis isolates. The heat map illustrates the distribution of core and accessory genes across the $G$. parasuis strains. The columns represent G. parasuis isolates. The rows represent genes. The red and black regions represent the presence or absence of genes in a particular genome, respectively. The black regions indicate features missing in that strain but present in one or more of the other $G$. parasuis strains. (C) The distribution of all, core, and specific genes according to the COG classification. The $y$-axis indicates the percentage of genes in various COG categories. A: RNAprocessing and modification. C: Energy production and conversion. D: Cell cycle control, cell division, chromosome partitioning. E:Amino acid transport and metabolism. F: Nucleotide transport and metabolism. G: Carbohydrate transport and metabolism. $\mathrm{H}$ : Coenzyme transport and metabolism. I: Lipid transport and metabolism. J: Translation, ribosomal structure and biogenesis. K: Transcription. L: Replication, recombination and repair. M: Cell wall/membrane/envelope biogenesis. N: Cell motility. O: Posttranslational modification, protein turnover, chaperones. P: Inorganic ion transport and metabolism. Q: Secondary metabolites biosynthesis, transport and catabolism. R: General function prediction only. S: Function unknown. T: Signal transduction mechanisms. U: Intracellular trafficking, secretion, and vesicular transport. V: Defense mechanisms. 

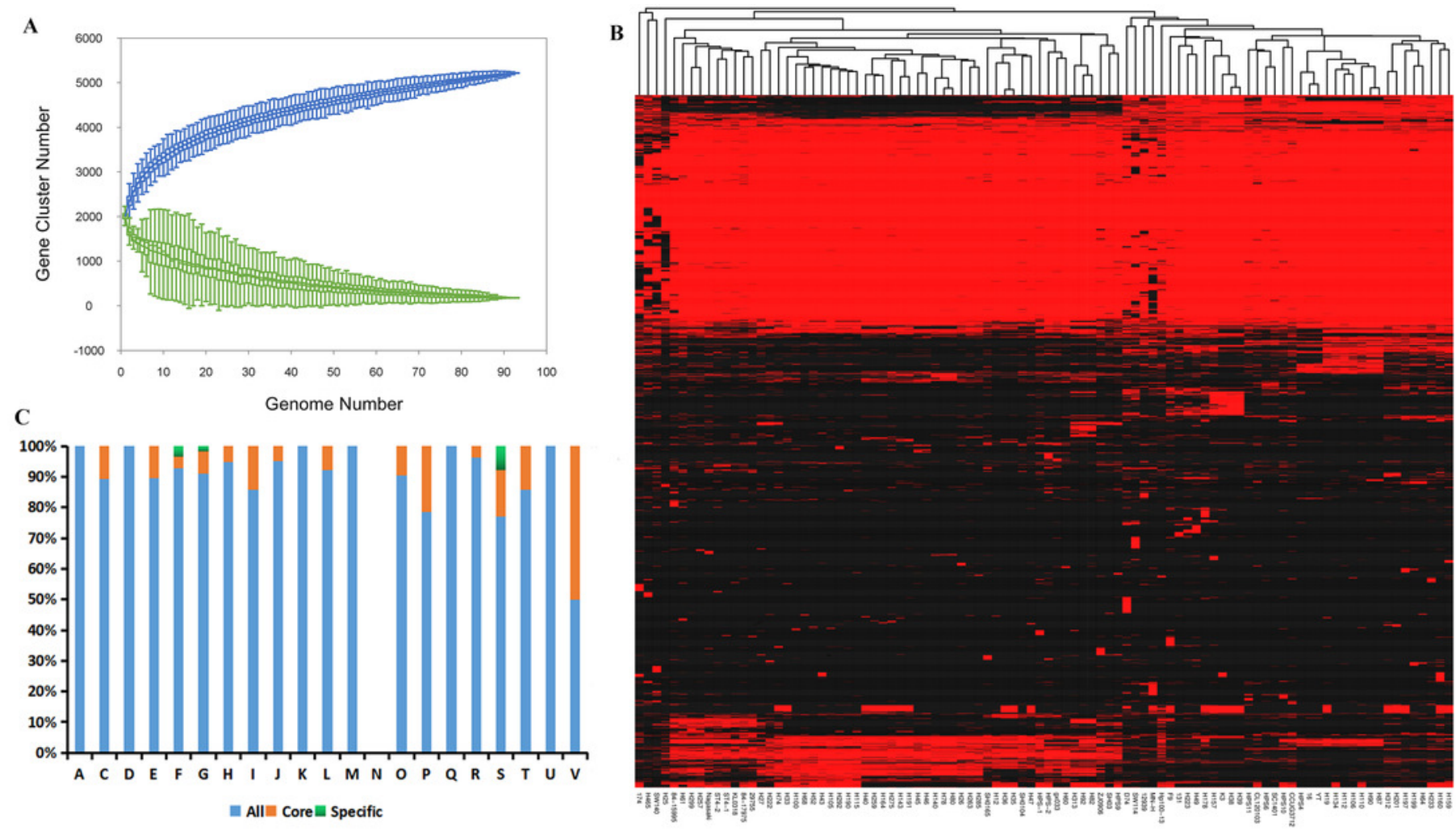
Figure 2

Maximum-likelihood phylogeny of 94 Glaesserella parasuis isolates based on 93 singlecopy core genes.

The tree was constructed with MEGA 7 with 1,000 bootstrap replicates. The annotation rings surrounding the tree, from outside to inside, depict (1) geographic region, (2) year of sample collection, (3) site of sample, and (4) serotype. The different colors of the branches represent lineages, lineage I in pink and lineage II in green.

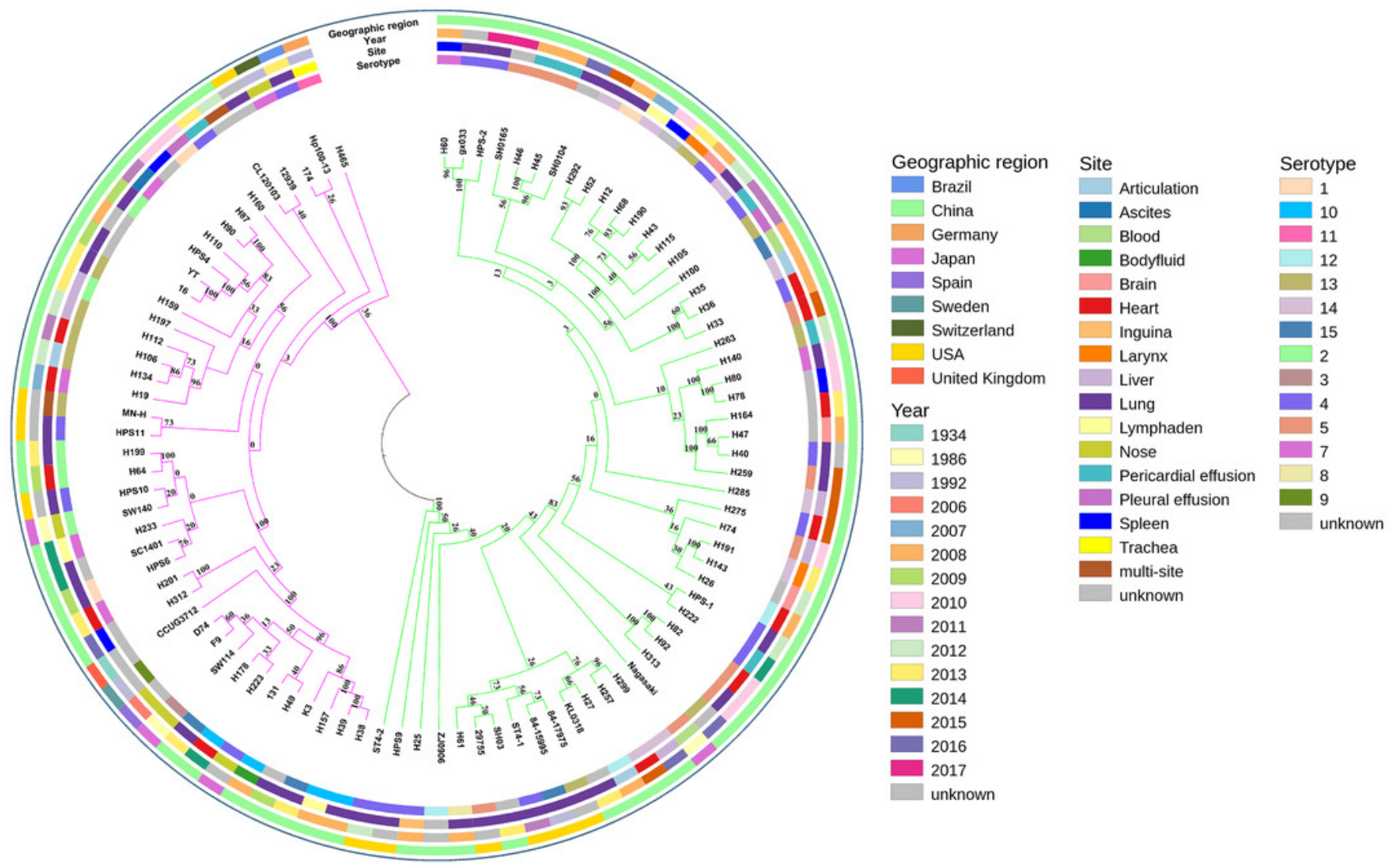




\section{Figure 3}

Virulence and resistance profiles across the phylogeny of the $94 \mathrm{G}$. parasuis isolates.

Cluster analysis based on single-copy core orthologs. Pattern of gene presence (colored line) or absence (white).

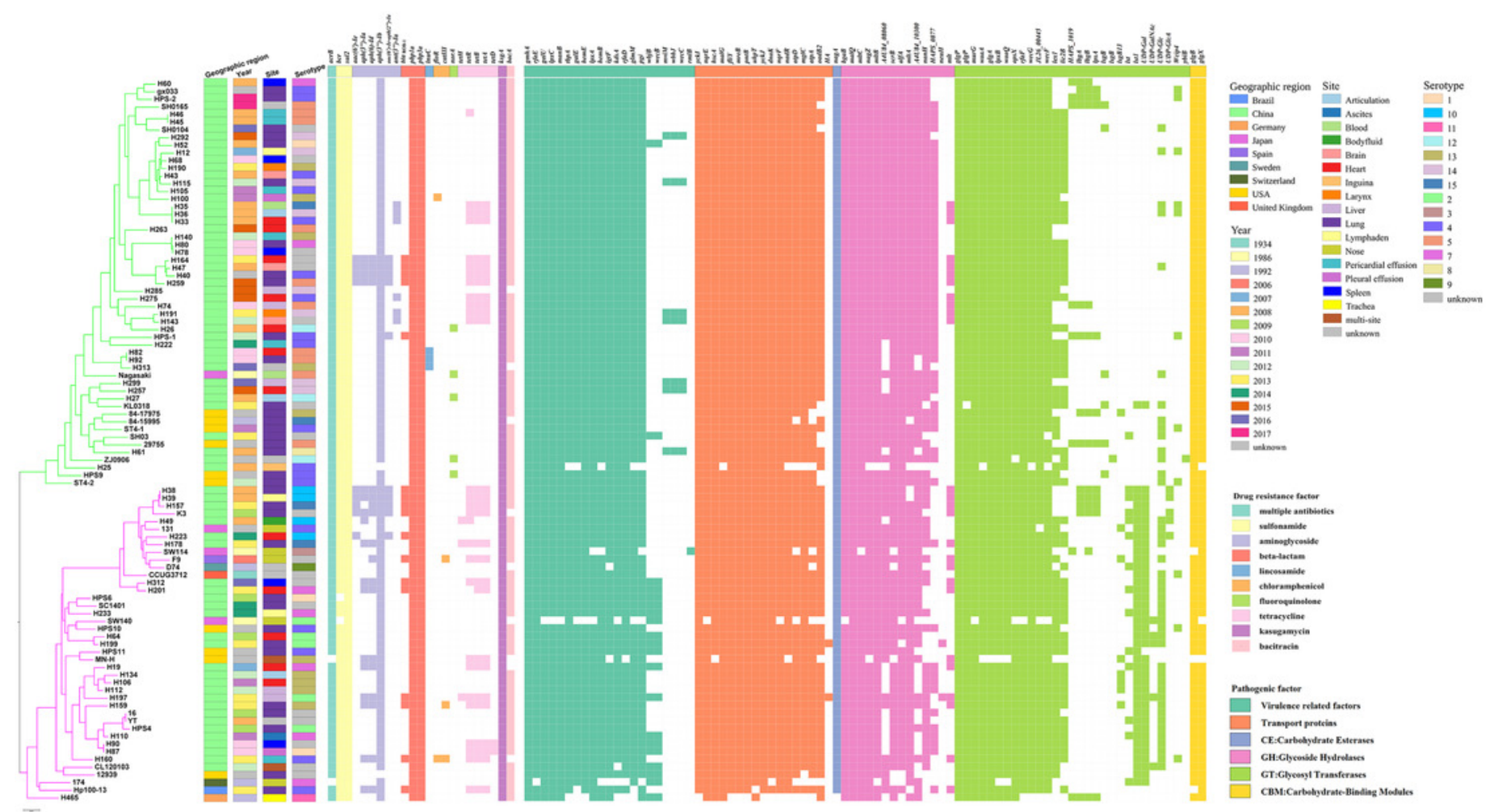


Figure 4

Schematic map of plasmid pYL1.

The circles show, from outside to inside: first and second, putative open reading frames, the positions and orientations of the genes; third, $\mathrm{G}+\mathrm{C}$ content (deviation from the average); and, fourth, G+C skew (green, +; purple, -).

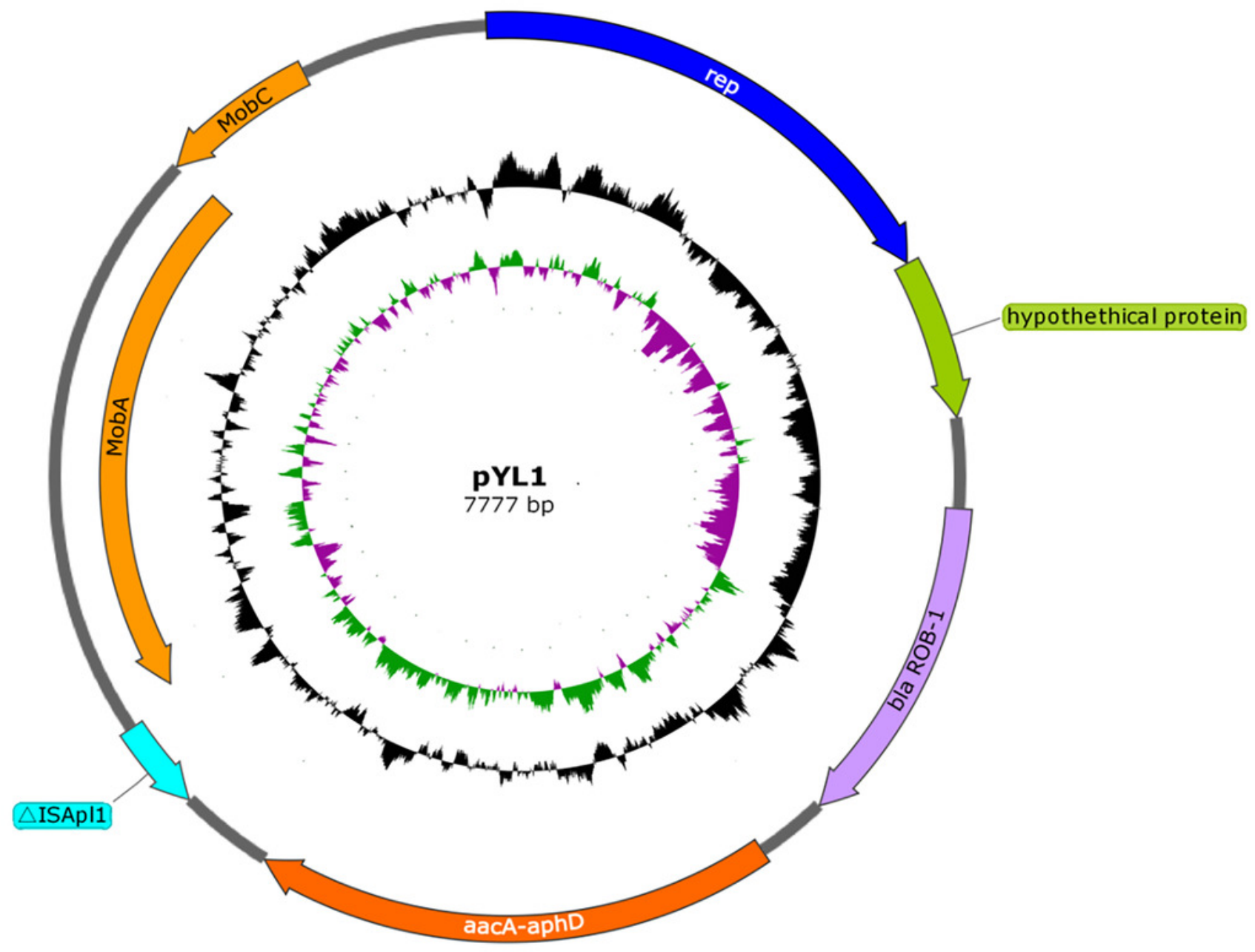




\section{Figure 5}

Organization of the G. parasuis HPS-1 Tn6678 transposon and comparison with the similar structure.

ORFs are shown as arrows, indicating the transcription direction, and the colors of the arrows represent different fragments. Gene color code: transposase, purple; toxin genes (pilT and $p h d)$, yellow; resistance genes( $c p x A, c p x R$ and $b c r)$, blue; proteins with other or unknown functions, gray. Homologous gene clusters in different isolates are shaded in gray (>97\%).

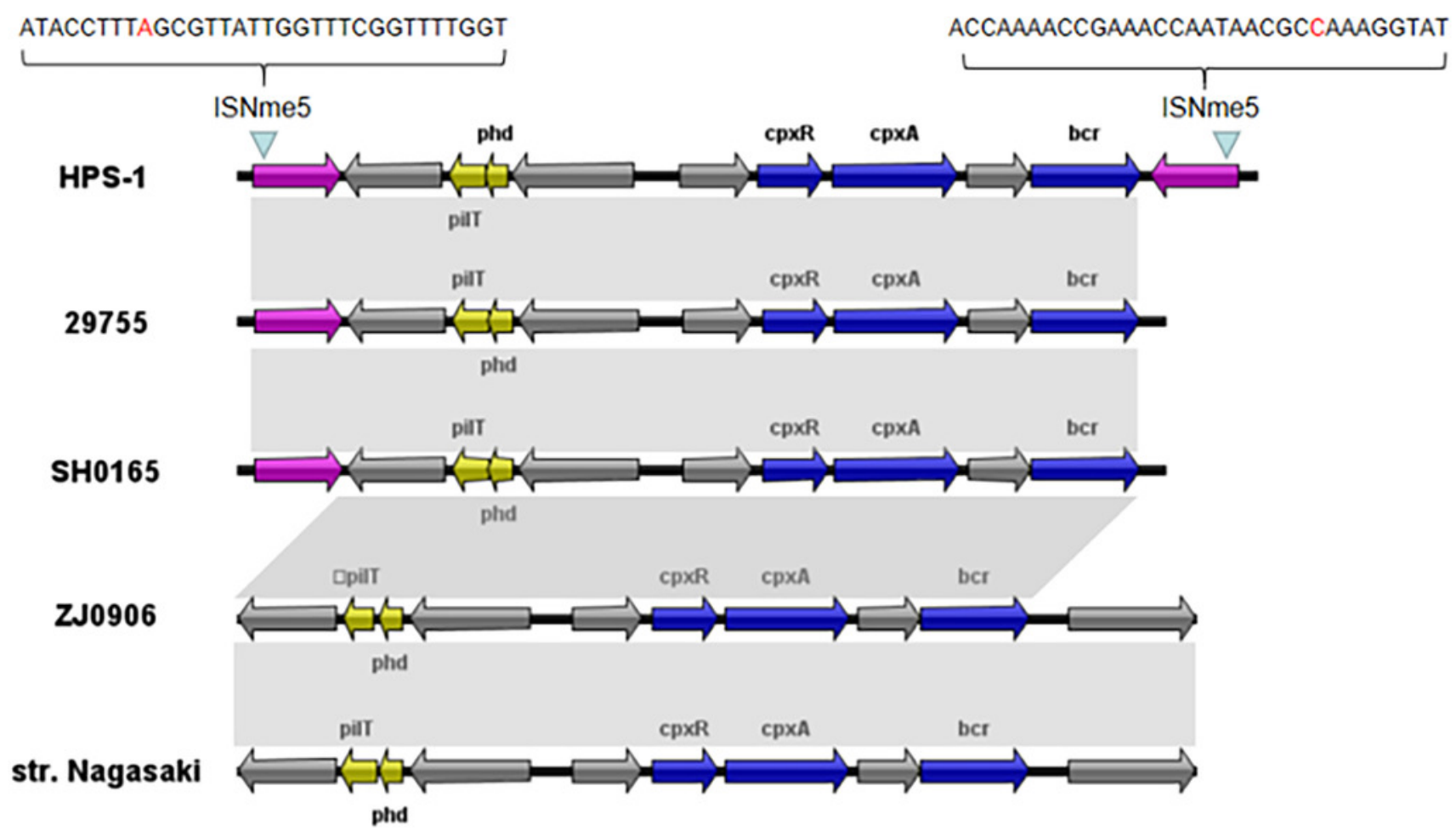


Figure 6

Neighbor-joining phylogenetic tree based on bcr gene sequences obtained from the current study and downloaded from NCBI.

The tree was constructed using MEGA 7 with 1,000 bootstrap replicates. The different colors of the branches represent lineages. The G. parasuis HPS-1 is indicated by a solid circle. 


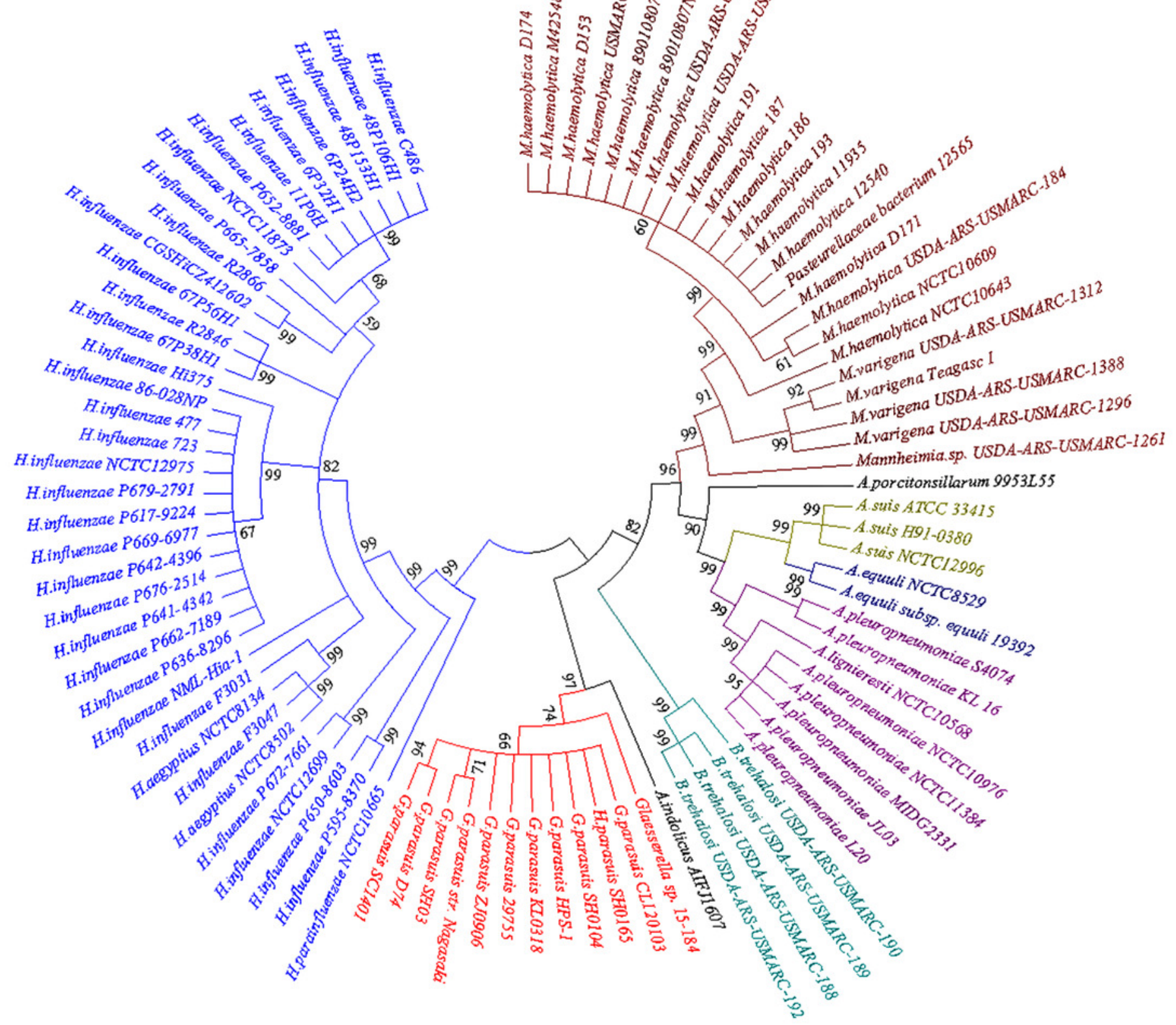




\section{Figure 7}

Comparison of the genetic structures of pHN61, pFS39, pYL1, pFZ51 and pHB0503.

The accession numbers and origins of these plasmids are displayed on the left side. Arrows represent putative open reading frames, the positions and orientations of the genes. Blue arrows indicate Rep-like protein involved in plasmid replication. Green arrows indicate hypothetical protein. Regions with more than $98 \%$ nucleotide sequence identity are shaded yellow.

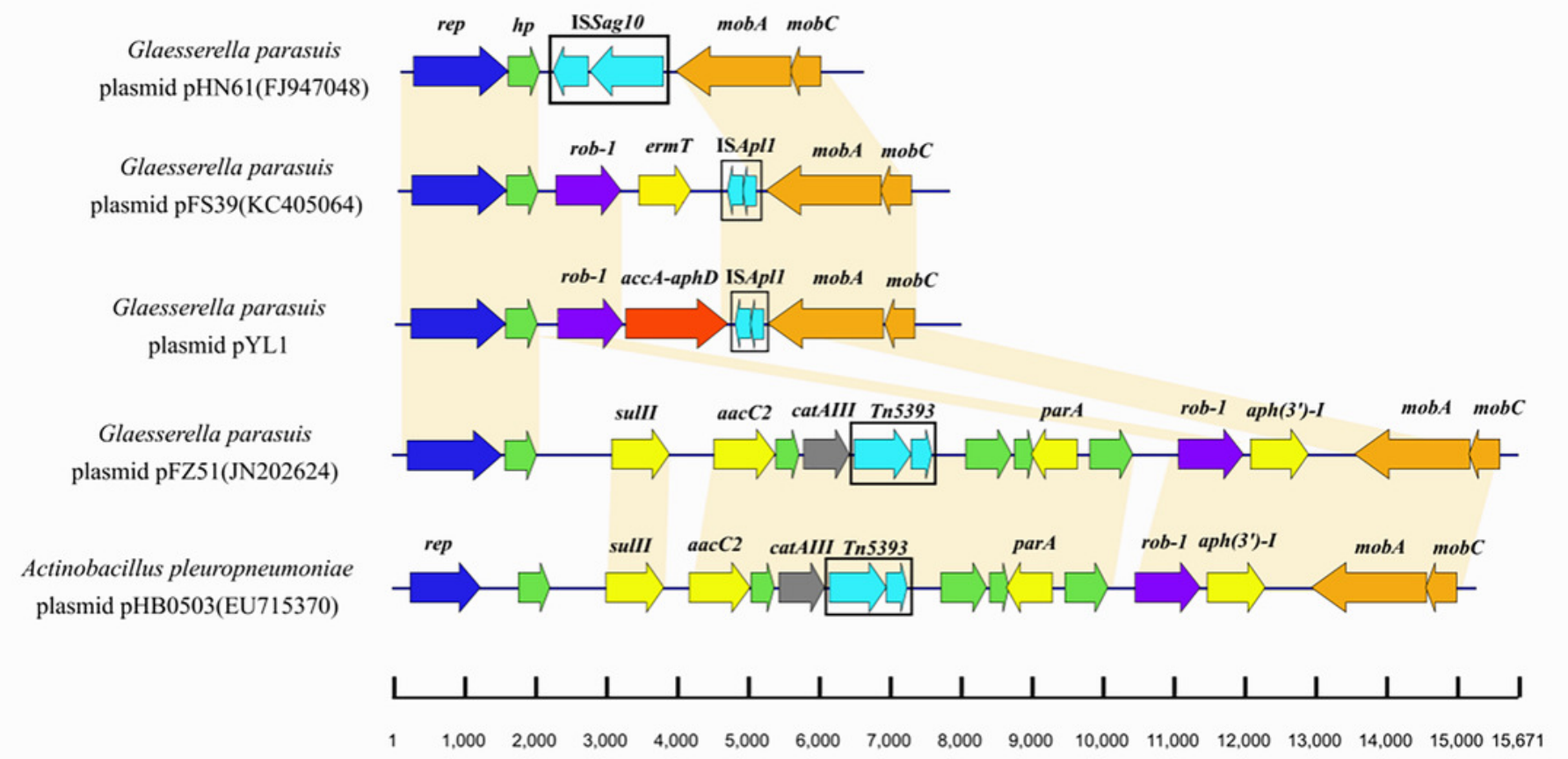

